\title{
Parallel Matrix Multiplication on a Linear Array with a Reconfigurable Pipelined Bus System
}

\author{
Keqin $\mathrm{Li}^{*}$ \\ Dept. of Computer Science \\ State University of New York \\ New Paltz, New York 12561-2440 \\ li@mcs.newpaltz.edu
}

\author{
Victor Y. Pan \\ Dept. of Math \& Computer Sci. \\ Lehman College, CUNY \\ Bronx, New York 10468-1589 \\ VPAN@lcvax.lehman.cuny.edu
}

\begin{abstract}
The known fast sequential algorithms for multiplying two $N \times N$ matrices (over an arbitrary ring) have time complexity $O\left(N^{\alpha}\right)$, where $2<\alpha<3$. The current best value of $\alpha$ is less than 2.3755. We show that for all $1 \leq p \leq N^{\alpha}$, multiplying two $N \times N$ matrices can be performed on a $p$-processor linear array with a reconfigurable pipelined bus system (LARPBS) in

$$
O\left(\frac{N^{\alpha}}{p}+\left(\frac{N^{2}}{p^{2 / \alpha}}\right) \log p\right)
$$

time. This is currently the fastest parallelization of the best known sequential matrix multiplication algorithm on a distributed memory parallel system. In particular, for all $1 \leq p \leq N^{2.3755}$, multiplying two $N \times N$ matrices can be performed on a $p$-processor LARPBS in

$$
O\left(\frac{N^{2.3755}}{p}+\left(\frac{N^{2}}{p^{0.8419}}\right) \log p\right)
$$

time, and linear speedup can be achieved for $p$ as large as $O\left(N^{2.3755} /(\log N)^{6.3262}\right)$. Furthermore, multiplying two $N \times N$ matrices can be performed on an LARPBS with $O\left(N^{\alpha}\right)$ processors in $O(\log N)$ time. This compares favorably with the performance on a PRAM.

Keywords: Bilinear algorithm, cost-optimality, distributed memory system, linear array, matrix multiplication, optical pipelined bus, PRAM, reconfigurable system, speedup.
\end{abstract}

${ }^{*}$ Author for correspondence. Phone: (845) 257-3534. Fax: (845) 257-3996. 


\section{Introduction}

Many sequential algorithms have been proposed for matrix multiplication, one of the most fundamental problems in sciences and engineering. The standard sequential algorithm takes $O\left(N^{3}\right)$ operations to multiply two $N \times N$ matrices. Since Strassen's remarkable discovery of his $O\left(N^{2.8074}\right)$ algorithm [35], successive progress has been made to develop fast sequential matrix multiplication algorithms with time complexity $O\left(N^{\alpha}\right)$, where $2<\alpha<3$. The current best exponent is $\alpha<2.3755$ [6].

The standard algorithm for matrix multiplication, Strassen's algorithm of [35] and Winograd's algorithm (see [27, page 169]) both with $\alpha<2.8074$, are used extensively in practice [9]. Other known asymptotically fast algorithms are not practically useful because of large overhead constants hidden in the big- $O$ notation (this applies to all the known algorithms with $\alpha<2.78$ ), or because of the requirement of increasing memory space [12]. It is still plausible that faster practical algorithms for matrix multiplication will appear, which should motivate theoretical importance of parallelization of matrix multiplication algorithms, and in the case of Strassen's and Winograd's algorithms, such a parallelization should already have practical value.

On shared memory multiprocessors, Strassen's algorithm has been parallelized [4]. Furthermore, it is well known that two $N \times N$ matrices can be multiplied under a CREW PRAM in $O(\log N)$ time by using $O\left(N^{\alpha+\varepsilon}\right)$ processors, for any fixed positive $\varepsilon$, as soon as we have an algorithm using $O\left(N^{\alpha}\right)$ arithmetic time for $N \times N$ matrix multiplication $[3,28,30]$. As a matter of fact, based on any of the known algorithms for $N \times N$ matrix multiplication running in $O\left(N^{\alpha}\right)$ time, we yield $O(\log N)$ parallel time using $O\left(N^{\alpha} / \log N\right)$ arithmetic processors under the PRAM model.

On distributed memory multicomputers (which are considered more practical), research has essentially focused on the parallelization of the standard method. It was shown that matrix multiplication can be done in $O\left(N^{3} / p+\log \left(p / N^{2}\right)\right)$ time on a hypercube with $p$ processors, where $N^{2} \leq p \leq N^{3}$ [7]. It was also reported that matrix multiplication can be done in constant time on a reconfigurable mesh with $N^{4}$ processors [31]. Such an implementation, though very fast, is far from cost-optimal.

To have fast and processor efficient parallel algorithms, it is necessary to consider non-standard algorithms. To the best of the authors' knowledge, all $O\left(N^{\alpha}\right)$ sequential algorithms with $\alpha<3$ have not been fully parallelized on any distributed memory systems, since these systems do not have sufficient capability to support complicated communication efficiently. In [7], it was shown that matrix multiplication can be done in $O\left(N^{\alpha} / p^{(\alpha-1) / 2}\right)$ time on a hypercube with $p$ processors, where $1 \leq p \leq N^{2}$. This algorithm is valid only in a small interval of $p$, which is not cost-optimal (as compared to the $O\left(N^{\alpha}\right)$ sequential algorithm), and the shortest execution time reached when $p=N^{2}$ is $O(N)$, which is very slow. The reason is that the $O\left(N^{\alpha}\right)$ algorithm is invoked sequentially to calculate submatrix products and not parallelized at all. 
To fully parallelize the fast sequential matrix multiplication algorithms on distributed memory systems, more powerful communication mechanism is required. It is clear that all existing realistic static networks with electronic connections have limited communication capability in supporting fast parallelization of an $O\left(N^{\alpha}\right)$ algorithm, where $\alpha<3$.

Recently, fiber optical buses have emerged as promising networks $[2,5,8,10,13,22,25$, 33, 38]. Pipelined optical buses can support massive volume of data transfer simultaneously, and can implement various communication patterns. Furthermore, a system with optical buses can be reconfigured into independent subsystems, which can be used simultaneously to solve subproblems in parallel [25]. It is now feasible to build distributed memory systems that are no less powerful and flexible than shared memory systems in solving many problems, such as Boolean matrix multiplication [14] and sorting [21]. Numerous parallel algorithms using optical interconnection networks have been developed recently $[1,11,17,18,19,20$, $23,24,26,32,34,36,37]$.

On a linear array with a reconfigurable pipelined bus system (LARPBS) proposed in [25], Strassen's algorithm has been parallelized and has execution time $O\left((\log N)^{\delta}\right)$ by using

$$
O\left(\frac{N^{3}}{1.1428^{(\log N)^{\delta}}}\right)
$$

processors, where $0 \leq \delta \leq 1$ [19]. This implies that matrix multiplication can be done in $O(1)$ time using $N^{3}$ processors, and in $O(\log N)$ time using $O\left(N^{2.8074}\right)$ processors. This is thus far the fastest parallel matrix multiplication algorithm on distributed memory models.

In this paper, we show that for all $1 \leq p \leq N^{\alpha}$, multiplying two $N \times N$ matrices (over an arbitrary ring) can be performed on a $p$-processor LARPBS in

$$
O\left(\frac{N^{\alpha}}{p}+\left(\frac{N^{2}}{p^{2 / \alpha}}\right) \log p\right)
$$

time. If the number of processors is

$$
p=O\left(\frac{N^{\alpha}}{(\log N)^{\alpha /(\alpha-2)}}\right),
$$

our algorithm achieves linear speedup and is cost-optimal. This is currently the fastest and most processor efficient parallelization of the best known sequential matrix multiplication algorithms on a distributed memory parallel system. In particular, for all $1 \leq p \leq N^{2.3755}$, multiplying two $N \times N$ matrices can be performed on a $p$-processor LARPBS in

$$
O\left(\frac{N^{2.3755}}{p}+\left(\frac{N^{2}}{p^{0.8419}}\right) \log p\right)
$$

time, and linear speedup can be achieved for $p$ as large as $O\left(N^{2.3755} /(\log N)^{6.3262}\right)$. Furthermore, multiplying two $N \times N$ matrices can be performed by an LARPBS with $O\left(N^{\alpha}\right)$ processors in $O(\log N)$ time. This matches the performance of PRAM. Also, it is clear that the processor complexity is substantially reduced as compared with that in [19]. 
The rest of the paper is organized as follows. In Section 2, we present the LARPBS computing model. Section 3 reviews the class of bilinear algorithms, and the strategy of their parallelization. In Section 4, we provide the implementation details of the fastest sequential matrix multiplication algorithm on an LARPBS, assuming that we have sufficient processors. Section 5 considers the case where the number of processors $p$ is arbitrarily chosen in the range $1 \leq p \leq N^{\alpha}$. In Section 6, we analyze the cost-optimality of our parallelization. We conclude the paper by raising an interesting open problem in Section 7.

\section{The LARPBS Computing Model}

A pipelined optical bus system uses optical waveguides instead of electrical signals to transfer messages among electronic processors. In addition to the high propagation speed of light, there are two important properties of optical signal (pulse) transmission on an optical bus, namely, unidirectional propagation and predictable propagation delay. These advantages of using waveguides enable synchronized concurrent accesses of an optical bus in a pipelined fashion [5, 13, 33]. Such pipelined optical bus systems can support a massive volume of communications simultaneously, and are particularly appropriate for applications that involve intensive communication operations such as broadcasting, one-to-one communication, multicasting, global aggregation, and irregular communication patterns.

A linear array with a reconfigurable pipelined bus system (LARPBS) consists of $N$ processors $P_{1}, P_{2}, P_{3}, \ldots, P_{N}$, connected by an optical bus. In addition to the tremendous communication capabilities, an LARPBS can also be partitioned into $k \geq 2$ independent

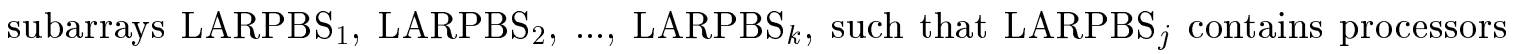
$P_{i_{j-1}+1}, P_{i_{j-1}+2}, \ldots, P_{i_{j}}$, where $0=i_{0}<i_{1}<i_{2} \cdots<i_{k}=N$. The subarrays can operate as regular linear arrays with pipelined optical bus systems, and all subarrays can be used independently for different computations without interference (see [25] for an elaborated exposition).

As in many other parallel computing systems, a computation on LARPBS is a sequence of alternate global communication and local computation steps. The time complexity of an algorithm is measured in terms of the total number of bus cycles in all the communication steps, as long as the time of the local computation steps between successive communication steps is bounded by a constant and independent of the problem size.

Perhaps the best way to understand the LARPBS computing model is to inspect the primitive operations that it can efficiently support. A number of basic communication, data movement, and aggregation operations on the LARPBS model implemented using the coincident pulse processor addressing technique [5, 13, 33] have been developed [19, 25]. Each of these primitive operations can be performed in a constant number of bus cycles. They provide an algorithmic view on parallel computing using optical interconnections, and also allow us to develop, specify, and analyze parallel algorithms by ignoring optical and engineering details. These powerful primitives that support massive parallel communications, plus the reconfigurability of the LARPBS model, make the LARPBS very attractive 
in solving problems that are both computation and communication intensive.

The following primitive operations on LARPBS are used in this paper, and our algorithms are developed using these operations as building blocks. The reader is referred to $[19,25]$ for the implementation details of these operations via optical signals. Here, we only give the definitions.

One-to-One Communication. Assume that processors $P_{i_{1}}, P_{i_{2}}, \ldots, P_{i_{q}}$ are senders, and processors $P_{j_{1}}, P_{j_{2}}, \ldots, P_{j_{q}}$ are receivers. In particular, processor $P_{i_{k}}$ sends a value $x_{i_{k}}$ to $P_{j_{k}}$, for all $1 \leq k \leq q$ simultaneously.

Broadcasting. Here, we have a source processor $P_{i}$, who sends a value $x$ to all the $N$ processors $P_{1}, P_{2}, P_{3}, \ldots, P_{N}$.

Multicasting. In a multicasting operation, we have a source processor $P_{i}$, who sends a value $x$ to a subset of the $N$ processors $P_{j_{1}}, P_{j_{2}}, \ldots, P_{j_{m}}$.

Multiple Multicasting. Assume that we have $g$ disjoint groups of destination processors, $G_{k}=\left\{P_{j_{k, 1}}, P_{j_{k, 2}}, P_{j_{k, 3}}, \ldots\right\}, 1 \leq k \leq g$, and there are $g$ senders $P_{i_{1}}, P_{i_{2}}, \ldots, P_{i_{g}}$. Processor $P_{i_{k}}$ has value $x_{i_{k}}$ to be broadcast to all the processors in $G_{k}$, where $1 \leq$ $k \leq g$. Since there are $g$ simultaneous multicasting, we have a multiple multicasting operation.

Global Summation. Suppose that every processor $P_{j}$ holds a numerical value $v_{j}, 1 \leq j \leq$ $N$, where $v_{j}$ is an integer or a floating-point value with finite magnitude and precision, we need to calculate the summation $v_{1}+v_{2}+v_{3}+\cdots+v_{N}$. The summation is finally saved in $P_{1}$.

All these communication, data movement, and global aggregation primitives can be performed on an LARPBS in constant number of bus cycles [19, 25].

It has been a common practice in algorithm analysis to assume that a single manipulation (e.g., an arithmetic operation) takes constant time. This essentially implies that numerical values have finite magnitude and precision; otherwise, a manipulation either takes longer time, or requires extra hardware support. Therefore, our assumption in the global summation is quite reasonable. However, since in this paper, we are dealing with matrix multiplication on an arbitrary ring, we need the following general aggregation operation.

Global Aggregation. Suppose that every processor $P_{j}$ holds a value $v_{j}, 1 \leq j \leq N$, where $v_{j}$ is in an arbitrary set $S$ with a binary associative operator $\oplus$, we need to calculate $v_{1} \oplus v_{2} \oplus v_{3} \oplus \cdots \oplus v_{N}$. The result of the aggregation is finally saved in $P_{1}$. We say that the size of the aggregation is $N$.

It may not be the case that all such kind of global aggregations can be implemented using optical signals in constant number of bus cycles. However, we can still use the ordinary binary tree method to find the aggregation in $O(\log N)$ time, where the data 
communications can be easily supported by an optical bus. If $N$ is a constant, such an aggregation requires constant amount of time. Fortunately, in this paper, we only use aggregations of constant sizes on subarrays whose sizes are independent of matrix sizes, and hence, their constant execution time is independent of the data set $S$ and the definition of $\oplus$. We will mention the size of each aggregation explicitly.

\section{The Strategy}

In this section, we show how to parallelize any fast sequential matrix multiplication algorithm, with one limitation, namely, the execution time is bounded from below by $O(\log N)$. This limitation is due to the recursive nature of the class of bilinear algorithms, not to the communication constraints on a parallel system. Assume that the known sequential algorithm for multiplying two $N \times N$ matrices has time complexity $O\left(N^{\alpha}\right)$.

We follow the approach [3, pages 315-316] and [28, 29, 30]. With no loss of generality, we will consider the class of recursive bilinear algorithms for the evaluation of the matrix product $C=A \times B$, where $A=\left(a_{i j}\right), B=\left(b_{j k}\right)$, and $C=\left(c_{i k}\right)$ are $m \times m$ matrices. First of all, there is a basis bilinear computation that has three steps.

Step (a). In the first step, the values of $2 R$ linear functions are computed,

$$
L_{u}=\sum_{1 \leq i, j \leq m} f(i, j, u) a_{i j}, \quad L_{u}^{*}=\sum_{1 \leq j, k \leq m} f^{*}(j, k, u) b_{j k},
$$

where $u=1,2, \ldots, R$.

Step (b). Then, in the second step, we compute the $R$ products $L_{u} L_{u}^{*}$, for all $1 \leq u \leq R$.

Step (c). Finally, in the third step, we calculate the $m^{2}$ outputs,

$$
c_{i k}=\sum_{j=1}^{m} a_{i j} b_{j k}=\sum_{u=1}^{R} f^{* *}(k, i, u) L_{u} L_{u}^{*},
$$

for all $1 \leq i, k \leq m$.

In the above computation, all the $f(i, j, u)$ 's, $f^{*}(j, k, u)$ 's, and $f^{* *}(k, i, u)$ 's are constants. The value $R$ is called the rank of the algorithm. For any positive $\varepsilon$ and any existent or plausible algorithm for $N \times N$ matrix multiplication running in $O\left(N^{\alpha}\right)$ arithmetic time, we may fix a natural $m$ and a basis bilinear computation with $R \leq m^{\alpha+\varepsilon}$ [27]. For all the known algorithms for $N \times N$ matrix multiplication running in $O\left(N^{\alpha}\right)$ time for $\alpha \leq 3$ (including the standard algorithm for $\alpha=3$, Strassen's algorithm for $\alpha=\log _{2} 7<2.8074$ [35], and the ones of [6] for $\alpha<2.3755$, which are currently asymptotically fastest), we have $R=m^{\alpha}$ in the associated basis bilinear construction. In the latter case, we will call $\alpha$ a basic exponent. (Note that for fixed $m$ and $\alpha, R$ is finite.) 
The above bilinear algorithm can be used recursively. Let $A=\left(A_{i j}\right), B=\left(B_{j k}\right)$, and $C=\left(C_{i k}\right)$ be $N \times N$ matrices, where $N=m^{n}$. Assume that $m$ is fixed, and $n \rightarrow \infty$. Each of these matrices are partitioned into $m^{2}$ submatrices $A_{i j}, B_{j k}, C_{i k}$ of size $m^{n-1} \times m^{n-1}$. Then, the above computation is still applicable when all the $a_{i j}$ 's, $b_{j k}$ 's, and $c_{i k}$ 's are replaced by submatrices. The recursive algorithm that computes $C=A \times B$ is described below.

Step (0). If the matrices are of size $m \times m$, compute the product $C=A \times B$ directly using the method above (i.e., Steps (a)-(c)), and return; otherwise, do Steps (1)-(3).

Step (1). Calculate $2 R$ linear combinations of submatrices

$$
L_{u}=\sum_{1 \leq i, j \leq m} f(i, j, u) A_{i j}
$$

and

$$
L_{u}^{*}=\sum_{1 \leq j, k \leq m} f^{*}(j, k, u) B_{j k},
$$

for all $1 \leq u \leq R$, where $L_{u}$ and $L_{u}^{*}$ are $m^{n-1} \times m^{n-1}$ matrices.

Step (2). Calculate the $R$ matrix products $L_{u} \times L_{u}^{*}$, for all $1 \leq u \leq R$.

Step (3). Compute

$$
C_{i k}=\sum_{j=1}^{m} A_{i j} B_{j k}=\sum_{u=1}^{R} f^{* *}(k, i, u)\left(L_{u} \times L_{u}^{*}\right),
$$

for all $1 \leq i, k \leq m$.

The above recursive algorithm has $n=\left\lceil\log _{m} N\right\rceil$ levels. (A few extra dummy rows and columns are introduced if $N$ is not a power of $m$.) The recursion reaches its base case (see Step (0)) when the size of the submatrices is $m \times m$. If sufficiently many processors are available, we can calculate the $L_{u}$ 's in parallel, and then all the $L_{u}^{*}$ 's in parallel (cf. Step (1)). After the recursion in Step (2), all the $C_{i k}$ 's are also computed in parallel (see Step (3)). Hence, each level takes constant time, and the overall time complexity is $O(\log N)$.

Under the PRAM model, the above computation only needs $R^{n} / \log N \leq m^{(\alpha+\varepsilon) n} / \log N$ $=N^{\alpha+\varepsilon} / \log N$ processors, for any fixed positive $\varepsilon$. Furthermore, for all so far available sequential algorithms for matrix multiplication, there is a parallelization which requires $O\left(N^{\alpha} / \log N\right)$ processors (see p.317 in [3]).

\section{Implementation Details}

In this section, we examine the implementation details of a bilinear algorithm on an LARPBS with $m^{2} R^{n}$ processors. Our algorithm is called Fast $(n)$, which stands for the fastest bilinear algorithm parallelized on an LARPBS with $p_{n}=m^{2} R^{n}$ processors $P_{1}, P_{2}$, $\ldots, P_{p_{n}}$, where $p_{n}$ is the total number of processors required in the above bilinear algorithm. 
The number of processors required to achieve the above maximum parallelism is analyzed as follows. When $n=1$, we have the base case. Step (a) needs $m^{2} R$ processors so that all the $L_{u}$ 's (and then all the $L_{u}^{*}$ 's) are obtained in parallel. Step (b) only requires $R$ processors. Step (c) takes $m^{2} R$ processors so that all the $c_{i k}$ 's are calculated in parallel. Hence, $p_{1}=m^{2} R$.

In general, when $n>1$, Steps (1) and (3) require $m^{2} R\left(m^{n-1}\right)^{2}=m^{2 n} R$ processors, and Step (2) needs $p_{n-1} R$ processors. That is, $p_{n}=\max \left(m^{2 n} R, p_{n-1} R\right)$. It can be proven by induction on $n$ that $p_{n}=m^{2} R^{n}$, for all $n \geq 1$. Therefore, for a fixed $m$, and $n \rightarrow \infty$, the total number of processors used for multiplying two $N \times N$ matrices, where $N=m^{n}$, is $p_{n}=O\left(R^{n}\right)=O\left(R^{\log _{m} N}\right)=O\left(N^{\log _{m} R}\right)=O\left(N^{\alpha}\right)$.

\subsection{The Base Case}

We first examine Fast(1), that is, the base case when $n=1$, which calculates $C=A \times B$, where $A=\left(a_{i j}\right), B=\left(b_{j k}\right)$, and $C=\left(c_{i k}\right)$ are $m \times m$ matrices, in constant time by using $p_{1}=m^{2} R$ processors.

Assume that initially, the input matrices $A$ and $B$ are stored in the first $m^{2}$ processors in the row-major order, that is, elements $a_{i j}$ and $b_{i j}$ are stored in processor $P_{(i-1) m+j}$, for all $1 \leq i, j \leq m$, and when algorithm Fast(1) completes, the output matrix $C$ is stored in the first $m^{2}$ processors in the row-major order, that is, element $c_{i j}$ is found in processor $P_{(i-1) m+j}$, for all $1 \leq i, j \leq m$,

For convenience, different index systems are used for the $p_{1}$ processors during the execution of algorithm Fast(1). In Step (a), processors are also named as $P_{u, i, j}$, where $1 \leq u \leq R$, and $1 \leq i, j \leq m$, and the processors are mapped to the linear array using the lexicographical order, namely, $P_{u, i, j}$ corresponds to $P_{(u-1) m^{2}+(i-1) m+j}$. We use $P_{u, *, *}$ to denote a subarray with $m^{2}$ processors, i.e., the $P_{u, i, j}$ 's for all $1 \leq i, j \leq m$. The $p_{1}$ processors will be divided into $R$ subarrays, $P_{1, *, *}, P_{2, *, *}, \ldots, P_{R, *, *}$, such that the subarray $P_{u, *, *}$ is used to calculate $L_{u}$ and $L_{u}^{*}$, where $1 \leq u \leq R$. There are three basic operations to compute the $L_{u}$ 's.

- For all $1 \leq i, j \leq m$, processor $P_{1, i, j}$ sends $a_{i j}$ to $P_{u, i, j}$, for all $2 \leq u \leq R$. This is actually a multiple multicasting operation. Thus, matrix $A$ is available to each subarray $P_{u, *, *}$, where $1 \leq u \leq R$.

- Processor $P_{u, i, j}$ performs one local calculation for $f(i, j, u) a_{i j}$, for all $1 \leq i, j \leq m$, and $1 \leq u \leq R$.

- The value $L_{u}$ is then calculated by the $m^{2}$ processors in $P_{u, *, *}$ via an aggregation of size $m^{2}$ for the summation $\sum_{1 \leq i, j \leq m} f(i, j, u) a_{i j}$. To this end, it is necessary to reconfigure the original LARPBS with $p_{1}$ processors into $R$ independent subsystems

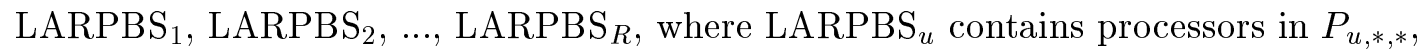
for all $1 \leq u \leq R$. 
The $L_{u}^{*}$ 's can be obtained in a similar way. Let us assume that after Step (a), $L_{u}$ and $L_{u}^{*}$ are hold by processor $P_{u, 1,1}$, for all $1 \leq u \leq R$.

In Step (b), there are two basic operations.

- Processor $P_{u, 1,1}$ sends $L_{u}$ and $L_{u}^{*}$ to processor $P_{u}$, for all $1 \leq u \leq R$ in parallel. This can be done using two one-to-one communications.

- Once processor $P_{u}$ receives $L_{u}$ and $L_{u}^{*}$, it can compute the product $L_{u} L_{u}^{*}$ using local computation.

After Step (b) is finished, processor $P_{u}$ has the values $L_{u} L_{u}^{*}$, for all $1 \leq u \leq R$.

In Step (c), processors are called $P_{i, k, u}$, where $1 \leq i, k \leq m$, and $1 \leq u \leq R$. The subarray $P_{i, k, *}$, which contains processors $P_{i, k, 1}, P_{i, k, 2}, \ldots, P_{i, k, R}$, is used to compute $c_{i k}$, for all $1 \leq i, k \leq m$.

- First, for all $1 \leq u \leq R$, processor $P_{u}$ sends the product $L_{u} L_{u}^{*}$ to processor $P_{i, k, u}$, for all $1 \leq i, k \leq m$, via a multiple multicasting operation.

- Second, processor $P_{i, k, u}$ computes the value $f^{* *}(k, i, u) L_{u} L_{u}^{*}$ locally.

- Third, the summation $c_{i k}=\sum_{u=1}^{R} f^{* *}(k, i, u) L_{u} L_{u}^{*}$ is assembled by the processors in $P_{i, k, *}$ using an aggregation operation of size $R$. The result $c_{i k}$ is then stored in $P_{i, k, 1}$, for all $1 \leq i, k \leq m$. System reconfiguration is required in this step.

- Finally, we need to perform a one-to-one communication to bring $c_{i k}$ from $P_{i, k, 1}$ to $P_{(i-1) m+k}$, for all $1 \leq i, k \leq m$, to meet the output data layout requirement.

It is clear that Steps (a), (b), and (c) can all be implemented using primitive data movement and communication operations, aggregations of constant sizes, and local operations, in constant number of bus cycles. Hence, we reach the following conclusion.

Theorem 1. Multiplying two $m \times m$ matrices can be performed in constant time on an LARPBS with $p_{1}=m^{2} R$ processors.

\subsection{The General Case}

Now, let us look at algorithm Fast $(n)$ in the general case where $n>1$. As mentioned before, the number of processors used by algorithm Fast $(n)$ is $p_{n}=m^{2} R^{n}$. Basically, we will show how each level of the recursion can be implemented in constant amount of time.

We assume that the input matrices $A$ and $B$, as well as the output matrix $C$ are all arranged in the shuffled-row-major order in the first $N^{2}$ processors of an LARPBS. Essentially, this means that if $A=\left(A_{i j}\right)$ of size $m^{n} \times m^{n}$ is divided into blocks $A_{i j}$ of size $m^{n-1} \times m^{n-1}$, then the blocks are arranged in the row-major order. Furthermore, the arrangement of the blocks are defined recursively, and the base matrices of size $m \times m$ are in the row-major order. 
In Step (0), we reach the base case. Therefore, algorithm Fast(1) is executed in constant time using $p_{1}$ processors to multiply two base matrices of size $m \times m$.

In Step (1), only $p_{n}^{\prime}=N^{2} R=m^{2 n} R<m^{2} R^{n}=p_{n}$ processors are required. These $p_{n}^{\prime}$ processors are divided into $m^{2} R$ groups, and each group of $m^{2(n-1)}$ processors is called a super-processor, which can be used to store a block $A_{i j}$ in the shuffled-row-major order. Let us label these super-processors as $\mathcal{P}_{u, i, j}$, where $1 \leq u \leq R$, and $1 \leq i, j \leq m$. The subarray of super-processors $\mathcal{P}_{u, *, *}$ calculates matrices $L_{u}$ and $L_{u}^{\prime}$, for all $1 \leq u \leq R$. It is clear that in the shuffled-row-major order, super-processor $\mathcal{P}_{1, i, j}$ holds the block $A_{i j}$, for all $1 \leq i, j \leq m$.

- For all $1 \leq i, j \leq m$, super-processor $\mathcal{P}_{1, i, j}$ sends $A_{i j}$ to $\mathcal{P}_{u, i, j}$, for all $2 \leq u \leq R$. This is accomplished by a multiple multicasting operation. Matrix $A$ is then available to each subarray $\mathcal{P}_{u, *, *}$, where $1 \leq u \leq R$.

- Super-processor $\mathcal{P}_{u, i, j}$ computes $f(i, j, u) A_{i j}$ using local calculation, for all $1 \leq i, j \leq$ $m$, and $1 \leq u \leq R$.

- The matrix $L_{u}$ of size $m^{n-1} \times m^{n-1}$ is then calculated by the $m^{2}$ super-processors in $\mathcal{P}_{u, *, *}$. To this end, in addition to reconfiguration of the original LARPBS into $R$ independent subsystems of super-processors, it is necessary to perform data rearrangement within each subsystem, and to reconfigure each subsystem into $m^{2(n-1)}$ sub-subsystems, such that each sub-subsystem (with $\mathrm{m}^{2}$ processors) calculates one element in $L_{u}$. There are three operations to obtain the submatrix summation $\sum_{1 \leq i, j \leq m} f(i, j, u) A_{i j}$, a one-to-one communication to bring the corresponding submatrix elements together into sub-subsystems, parallel aggregations of size $m^{2}$ in the sub-subsystems, and a one-to-one communication to put the results to the right processors. Detailed specification is omitted here.

The matrix $L_{u}^{*}$ can be obtained in a similar way. Let us assume that after Step (1), $L_{u}$ and $L_{u}^{*}$ are hold by super-processor $\mathcal{P}_{u, 1,1}$ in the shuffled-row-major order, for all $1 \leq u \leq R$.

In Step (2), all the $p_{n}$ processors are employed. These $p_{n}$ processors will be divided into $R$ subsystems $\mathrm{LARPBS}_{1}, \mathrm{LARPBS}_{2}, \ldots, \mathrm{LARPBS}_{R}$, and each subsystem LARPBS has $p_{n-1}$ processors used to calculate the matrix product $L_{u} \times L_{u}^{*}$, for all $1 \leq u \leq R$. For convenience, processors are also grouped into super-processors.

- Super-processor $\mathcal{P}_{u, 1,1}$ sends $L_{u}$ and $L_{u}^{*}$ to the first super-processor in $\mathrm{LARPBS}_{u}$, for all $1 \leq u \leq R$ in parallel, using two one-to-one communications.

- $\mathrm{LARPBS}_{u}$ computes the product $L_{u} \times L_{u}^{*}$ by recursively invoking algorithm Fast $(n-1)$ and using $p_{n-1}$ processors, where $1 \leq u \leq R$.

After Step (2) is finished, the first super-processor in $\mathrm{LARPBS}_{u}$ has the matrices $L_{u} \times L_{u}^{*}$, for all $1 \leq u \leq R$. 
In Step (3), again, only $p_{n}^{\prime}$ processors are used. Once more, the $p_{n}^{\prime}$ processors are grouped into super-processors $\mathcal{P}_{i, k, u}$, where $1 \leq i, k \leq m$, and $1 \leq u \leq R$, and each super-processor has $m^{2(n-1)}$ processors. These $m^{2} R$ super-processors are divided into $m^{2}$ subarrays $\mathcal{P}_{i, k, *}$, and $\mathcal{P}_{i, k, *}$ is used to compute one submatrix $C_{i k}$, where $1 \leq i, k \leq m$. Each subarray $\mathcal{P}_{i, k, *}$ has $R$ super-processors $\mathcal{P}_{i, k, 1}, \mathcal{P}_{i, k, 2}, \ldots, \mathcal{P}_{i, k, R}$, where $\mathcal{P}_{i, k, u}$ holds the matrix product $L_{u} \times L_{u}^{*}$, for all $1 \leq u \leq R$.

- For all $1 \leq u \leq R$, the first super-processor in $\mathrm{LARPBS}_{u}$ of Step (2) sends the matrix product $L_{u} \times L_{u}^{*}$ to all $P_{i, k, u}$, where $1 \leq i, k \leq m$.

- Super-processor $\mathcal{P}_{i, k, u}$ calculates $f^{* *}(k, i, u)\left(L_{u} \times L_{u}^{*}\right)$ by local computation, where $1 \leq i, k \leq m$, and $1 \leq u \leq R$.

- Subarray $\mathcal{P}_{i, k, *}$ computes the submatrix summation $\sum_{u=1}^{R} f^{* *}(k, i, u)\left(L_{u} \times L_{u}^{*}\right)$, where $1 \leq i, k \leq m$, through appropriate data rearrangement and aggregations of size $R$. The summation is stored in super-processor $\mathcal{P}_{i, k, 1}$.

- Super-processor $\mathcal{P}_{i, k, 1}$ sends the result $C_{i k}$ to the right place via a one-to-one communication, for all $1 \leq i, k \leq m$.

It is clear that except the recursion in Step (2), all other steps take constant number of bus cycles. Since each of the $O(\log N)$ levels of the recursion requires constant amount of time, algorithm Fast $(n)$ has time complexity $O(\log N)$. Thus, we have the following claim.

Theorem 2. For any basic exponent $\alpha$, multiplying two $N \times N$ matrices can be performed in $O(\log N)$ time on an LARPBS with $p_{n}=O\left(N^{\alpha}\right)$ processors.

\section{$5 \quad$ Fewer Processors: $1 \leq \mathrm{p} \leq \mathbf{N}^{\alpha}$}

In the last section, we provide the implementation details of parallelization of the fastest sequential matrix multiplication algorithm when there are sufficiently many processors. In reality, the number of processors available is not always enough for an application. In this section, we examine the case where the number of processors $p$ is arbitrarily chosen in the range $\left[1 . . N^{\alpha}\right]$.

It is clear that in the implementation of Section 4, processors perform computation and communication at the element level, that is, processors

- send to each other one matrix element at a time;

- calculate element product locally;

- and aggregate element summations. 
(Remark: Communication and aggregation of submatrices among super-processors are actually done by individual processors at the element level.) All the above operations take constant bus cycles. When there are fewer processors, it is necessary for processors to perform computation and communication at the submatrix level.

Let $q$ be an integer such that $q^{\alpha} \leq p$, i.e., $q=\left\lfloor p^{1 / \alpha}\right\rfloor$, and $s=\lceil N / q\rceil$. All the matrices $A=\left(A_{i j}\right), B=\left(B_{j k}\right)$, and $C=\left(C_{i k}\right)$ are partitioned into submatrices $A_{i j}, B_{j k}, C_{i k}$ of size $s \times s$. Our algorithm is called Fast $(n, p)$, which is essentially the same as Fast $(n)$, except that we imagine that each of these submatrices is a single element. Therefore, processors

- send to each other one submatrix at a time;

- calculate submatrix product locally;

- and aggregate submatrix summations.

Each communication (i.e., one-to-one and multiple multicasting) or aggregation at the submatrix level can be realized by $s^{2}$ communication or aggregation at the element level. This implies that the execution times of Steps (1), (2), and (3) are augmented by a factor of $s^{2}$. In Step (0), i.e., the base case, each processor calculates submatrix multiplication sequentially, which takes $O\left(s^{\alpha}\right)$ time. It seems that the matrix sizes are reduced from $N \times N$ to $q \times q$, if all submatrices are treated as single elements. This implies that the number of levels of recursion is $\left\lceil\log _{m} q\right\rceil$. Therefore, the overall execution time $T(N, p, \alpha)$ of algorithm Fast $(n, p)$ to multiply two $N \times N$ matrices using $p$ processors by parallelizing the best $O\left(N^{\alpha}\right)$ sequential algorithm is

$$
\begin{aligned}
T(N, p, \alpha) & =O\left(s^{\alpha}+s^{2} \log q\right) \\
& =O\left(\left(\frac{N}{q}\right)^{\alpha}+\left(\frac{N}{q}\right)^{2} \log \left(p^{1 / \alpha}\right)\right) \\
& =O\left(\frac{N^{\alpha}}{p}+\frac{1}{\alpha}\left(\frac{N^{2}}{p^{2 / \alpha}}\right) \log p\right) .
\end{aligned}
$$

The above discussion essentially proves the following theorem.

Theorem 3. For any basic exponent $\alpha$ and for all $1 \leq p \leq N^{\alpha}$, multiplying two $N \times N$ matrices can be performed on a $p$-processor LARPBS in

$$
O\left(\frac{N^{\alpha}}{p}+\left(\frac{N^{2}}{p^{2 / \alpha}}\right) \log p\right)
$$

time.

Theorem 2 is a special case of Theorem 3 by taking $p=N^{\alpha}$.

Corollary 1. For any basic exponent $\alpha$, multiplying two $N \times N$ matrices can be performed on an LARPBS in $O(\log N)$ time, using $N^{\alpha}$ processors. In particular, multiplying two $N \times N$ matrices can be performed on an LARPBS in $O(\log N)$ time, using $N^{2.3755}$ processors.

Notice that Corollary 1 is well known on the PRAM model [30]. 


\section{Cost-Optimality}

We now show that algorithm Fast $(n, p)$ achieves linear speedup and is cost-optimal in a wide range of $p$ for all the known $N \times N$ matrix multiplication algorithms. It is clear from Theorem 3 that when $p \ll N$, the term $O\left(N^{\alpha} / p\right)$ dominates the time complexity of Fast $(n, p)$. When $p$ is close to $N^{\alpha}$, the second term dominates $T(N, p, \alpha)$. Since

$$
T(N, p, \alpha)=O\left(\frac{N^{\alpha}}{p}+\left(\frac{N^{\alpha}}{p}\right)^{2 / \alpha} \log p\right),
$$

we write $p=N^{\alpha} / f(N)$, which yields

$$
T(N, p, \alpha)=O\left(f(N)+(f(N))^{2 / \alpha} \log N\right),
$$

where $f(N)$ is a small infinity. Clearly, when $f(N)=O\left((\log N)^{\alpha /(\alpha-2)}\right)$, we have

$$
T(N, p, \alpha)=O\left((f(N))^{2 / \alpha} \log N\right) .
$$

When $p$ does not exceed $O\left(N^{\alpha} /(\log N)^{\alpha /(\alpha-2)}\right)$, the speedup of algorithm Fast $(n, p)$ is

$$
S(N, p, \alpha)=O(p) \text {. }
$$

When $p=\Omega\left(N^{\alpha} /(\log N)^{\alpha /(\alpha-2)}\right)$, we still have

$$
S(N, p, \alpha)=\frac{O\left(N^{\alpha}\right)}{T(N, p, \alpha)}=\Omega\left(\frac{N^{\alpha-2} p^{2 / \alpha}}{\log N}\right)=\Omega\left(\frac{p}{\log N}\right) .
$$

Similarly, the cost of algorithm Fast $(n, p)$ is

$$
C(N, p, \alpha)=O\left(N^{\alpha}\right)
$$

for $p$ as large as $O\left(N^{\alpha} /(\log N)^{\alpha /(\alpha-2)}\right)$. When $p=\Omega\left(N^{\alpha} /(\log N)^{\alpha /(\alpha-2)}\right)$, we have

$$
C(N, p, \alpha)=T(N, p, \alpha) p=O\left(p^{(\alpha-2) / \alpha} N^{2} \log N\right) .
$$

The highest cost is $O\left(N^{\alpha} \log N\right)$, when $p=N^{\alpha}$.

Corollary 2. If the number of processors is

$$
p=O\left(\frac{N^{\alpha}}{(\log N)^{\alpha /(\alpha-2)}}\right),
$$

algorithm Fast $(n, p)$ achieves linear speedup and is cost-optimal.

The following result is an immediate consequence of Theorem 3 and Corollary 2.

Corollary 3. For all $1 \leq p \leq N^{2.3755}$, multiplying two $N \times N$ matrices can be performed on a $p$-processor LARPBS in

$$
O\left(\frac{N^{2.3755}}{p}+\left(\frac{N^{2}}{p^{0.8419}}\right) \log p\right)
$$

time. Hence, linear speedup can be achieved for $p=O\left(N^{2.3755} /(\log N)^{6.3262}\right)$. 


\section{Concluding Remarks}

We have developed an efficient parallelization of the fastest sequential matrix multiplication algorithm on a linear array with a reconfigurable pipelined optical bus system. The algorithm has linear speedup and cost-optimality in a wide range of choice for the number of processors. It turns out that for parallel matrix multiplication, a distributed memory system with optical interconnections like LARPBS compares favorably with shared memory systems, where the concurrent read capability is replaced by highly efficient communications with predictable senders and receivers.

The results of this paper can be applied to solve many other matrix computation problems such as calculating matrix chain products, computing the powers, the characteristic polynomial, the determinant, the rank, the inverse, an LU-factorization, and a QR-factorization of a matrix, solving linear systems of equations, and so on. All these computations contain matrix multiplication as subcomputations, and hence fast and scalable parallel algorithms can be developed $[15,16]$.

As indicated in Section 3, under the PRAM model, $O\left(N^{\alpha} / \log N\right)$ processors are enough to achieve $O(\log N)$ parallel execution time. While such a parallelization is not difficult under the PRAM model, where all the processors share infinite common memory, it is more involved to implement such a parallelization in a distributed memory model. However, we believe that by carefully designing data distribution and scheduling the computation and communication, it is possible to parallelize a sequential $O\left(N^{\alpha}\right)$ matrix multiplication algorithm on an LARPBS in $O(\log N)$ time by using $O\left(N^{\alpha} / \log N\right)$ processors. This will be our next step of investigation.

It is also noticed that the time complexity of algorithm Fast $(n, p)$ is bounded from below by $O(\log N)$. An interesting question is, "Can we obtain sublogarithmic execution time by using more processors?" The following theorem was established in [19].

Theorem 4. For all $p=N^{3} /\left(\frac{8}{7}\right)^{(\log N)^{\delta}}$, where $0 \leq \delta \leq 1$, multiplying two $N \times N$ matrices can be performed on a $p$-processor LARPBS in $O\left((\log N)^{\delta}\right)$ time.

When $\delta<1$, we have $\left(\frac{8}{7}\right)^{(\log N)^{\delta}}=o\left(N^{\epsilon}\right)$, for all $\epsilon>0$. This means that Theorem 4 results in sublogarithmic time complexity for matrix multiplication in an interval of $p$ that is less than $\left[N^{3-\epsilon}, N^{3}\right]$, for all $\epsilon>0$. An interesting open problem is, "Can we obtain sublogarithmic execution time by using $N^{3-\epsilon}$ processors, for some $\epsilon>0$ ?" This is worth of further investigation. 


\section{Acknowledgments}

K. Li was supported by National Aeronautics and Space Administration and the State University of New York through the NASA/University Joint Venture (JOVE) in Space Science Program under Grant NAG8-1313. V.Y. Pan was supported by National Science Foundation under Grants CCR 9625344 and CCR 9732206, and PSC CUNY Awards 668365

and 669363. A preliminary version of the paper appeared in Proceedings of IPPS/SPDP '99 (Second Merged Symposium of 13th International Parallel Processing Symposium and 10th Symposium on Parallel and Distributed Processing), pp. 31-35, San Juan, Puerto Rico, April 1999.

\section{References}

[1] S.G. Akl, Parallel Computation: Models and Methods, Prentice-Hall, Upper Saddle River, New Jersey, 1997.

[2] A.F. Benner, H.F. Jordan, and V.P. Heuring, "Digital optical computing with optically switched directional couplers," Optical Engineering, vol. 30, pp. 1936-1941, 1991.

[3] D. Bini and V. Pan, Polynomial and Matrix Computations, Vol. 1, Fundamental Algorithms, Birkhäuser, Boston, 1994.

[4] A.K. Chandra, "Maximal parallelism in matrix multiplication," Report RC-6193, IBM T.J. Watson Research Center, Yorktown Heights, New York, October 1979.

[5] D. Chiarulli, R. Melhem, and S. Levitan, "Using coincident optical pulses for parallel memory addressing," IEEE Computer, vol. 30, pp. 48-57, 1987.

[6] D. Coppersmith and S. Winograd, "Matrix multiplication via arithmetic progressions," Journal of Symbolic Computation, vol. 9, pp. 251-280, 1990.

[7] E. Dekel, D. Nassimi, and S. Sahni, "Parallel matrix and graph algorithms," SIAM Journal on Computing, vol. 10, pp. 657-673, 1981.

[8] P.W. Dowd, "Wavelength division multiple access channel hypercube processor interconnection," IEEE Transactions on Computers, vol. 41, pp. 1223-1241, 1992.

[9] G.H. Golub and C.F. Van Loan, Matrix Computations, Johns Hopkins University Press, Baltimore, Maryland, 1996.

[10] Z. Guo, R. Melhem, R. Hall, D. Chiarulli, and S. Levitan, "Pipelined communications in optically interconnected arrays," Journal of Parallel and Distributed Computing, vol. 12, pp. 269-282, 1991.

[11] M. Hamdi and Y. Pan, "Efficient parallel algorithms on optically interconnected arrays of processors," IEE Proceedings - Computers and Digital Techniques, vol. 142, pp. 87-92, 1995.

[12] J. Laderman, V.Y. Pan, and X.-H. Sha, "On practical acceleration of matrix multiplication," Linear Algebra and Its Applications, vol. 162-164, pp. 557-588, 1992. 
[13] S. Levitan, D. Chiarulli, and R. Melhem, "Coincident pulse techniques for multiprocessor interconnection structures," Applied Optics, vol. 29, pp. 2024-2039, 1990.

[14] K. Li, "Constant time boolean matrix multiplication on a linear array with a reconfigurable pipelined bus system," Journal of Supercomputing, vol. 11, no. 4, pp. 391-403, 1997.

[15] K. Li, "Fast and scalable parallel algorithms for matrix chain product and matrix powers on optical buses," in High Performance Computing Systems and Applications, A. Pollard, D.J.K. Mewhort, and D.F. Weaver, eds., pp. 333-348, Kluwer Academic Publishers, Boston, Massachusetts, 2000.

[16] K. Li, "Fast and scalable parallel matrix computations with optical buses," Lecture Notes in Computer Science, vol. 1800, pp. 1053-1062, 2000.

[17] K. Li, Y. Pan, and M. Hamdi, "Solving graph theory problems using reconfigurable pipelined optical buses," Parallel Computing, vol. 26, no. 6, pp. 723-735, 2000.

[18] K. Li, Y. Pan, and S.Q. Zheng, eds., Parallel Computing Using Optical Interconnections, Kluwer Academic Publishers, Boston, Massachusetts, 1998.

[19] K. Li, Y. Pan, and S.Q. Zheng, "Fast and processor efficient parallel matrix multiplication algorithms on a linear array with a reconfigurable pipelined bus system," IEEE Transactions on Parallel and Distributed Systems, vol. 9, no. 8, pp. 705-720, 1998.

[20] K. Li, Y. Pan, and S.Q. Zheng, "Parallel matrix computations using a reconfigurable pipelined optical bus," Journal of Parallel and Distributed Computing, vol. 59, no. 1, pp. 13-30, 1999.

[21] K. Li, Y. Pan, and S.Q. Zheng, "Efficient deterministic and probabilistic simulations of PRAMs on linear arrays with reconfigurable pipelined bus systems," Journal of Supercomputing, vol. 15, no. 2, pp. 163-181, 2000.

[22] Y. Li, Y. Pan, and S.Q. Zheng, "Pipelined TDM optical bus with conditional delays," Optical Engineering, vol. 36, no. 9, pp. 2417-2424, 1997.

[23] Y. Pan and M. Hamdi, "Efficient computation of singular value decomposition on arrays with pipelined optical buses," Journal of Network and Computer Applications, vol. 19, pp. 235-248, 1996.

[24] Y. Pan, M. Hamdi, and K. Li, "Efficient and scalable quicksort on a linear array with a reconfigurable pipelined bus system," Future Generation Computer Systems, vol. 13, no. 6, pp. 501-513, 1998.

[25] Y. Pan and K. Li, "Linear array with a reconfigurable pipelined bus system - concepts and applications," Journal of Information Sciences, vol. 106, no. 3-4, pp. 237-258, 1998.

[26] Y. Pan, K. Li, and S.Q. Zheng, "Fast nearest neighbor algorithms on a linear array with a reconfigurable pipelined bus system," Journal of Parallel Algorithms and Applications, vol. 13, pp. 1-25, 1998.

[27] V. Pan, "How to multiply matrices faster," Lecture Notes in Computer Science, vol. 179, Springer Verlag, Berlin, 1984. 
[28] V. Pan, "Complexity of parallel matrix computations," Theoretical Computer Science, vol. 54, pp. 65-85, 1987.

[29] V. Pan, "Parallel solution of sparse linear and path systems," in Synthesis of Parallel Algorithms, J.H. Reif, ed., pp. 621-678, Morgan Kaufmann, San Mateo, California, 1993.

[30] V. Pan and J. Reif, "Efficient parallel solution of linear systems," Proceedings of "th ACM Symposium on Theory of Computing, pp. 143-152, May 1985.

[31] H. Park, H.J. Kim, and V.K. Prasanna, "An $O(1)$ time optimal algorithm for multiplying matrices on reconfigurable mesh," Information Processing Letters, vol. 47, pp. 109-113, 1993.

[32] S. Pavel and S.G. Akl, "Matrix operations using arrays with reconfigurable optical buses," Journal of Parallel Algorithms and Applications, vol. 8, pp. 223-242, 1996.

[33] C. Qiao and R. Melhem, "Time-division optical communications in multiprocessor arrays," IEEE Transactions on Computers, vol. 42, pp. 577-590, 1993.

[34] S. Rajasekaran and S. Sahni, "Sorting, selection, and routing on the array with reconfigurable optical buses," IEEE Transactions on Parallel and Distributed Systems, vol. 8, no. 11, pp. 1123-1132, 1997.

[35] V. Strassen, "Gaussian elimination is not optimal," Numerische Mathematik, vol. 13, pp. 354$356,1969$.

[36] J.L. Trahan, A.G. Bourgeois, Y. Pan, and R. Vaidyanathan, "Optimally scaling permutation routing on reconfigurable linear arrays with optical buses," Journal of Parallel and Distributed Computing, vol. 60, no. 9, pp. 1125-1136, 2000.

[37] C.-H. Wu, S.-J. Horng, and H.-R. Tsai, "Efficient parallel algorithms for hierarchical clustering on arrays with reconfigurable optical buses," Journal of Parallel and Distributed Computing, vol. 60 , no. 9, pp. 1137-1153, 2000.

[38] S.Q. Zheng and Y. Li, "Pipelined asynchronous time-division multiplexing optical bus," Optical Engineering, vol. 36, no. 12, pp. 3392-3400, 1997. 